with a smaller lesion.

Spiegel (1962), after investigating pallidofugal patheway, reported a new target called campotomy which involves the zona incerta and the Forel $\mathrm{H}$ field.

A subthalamotomy in which the subthalamic area is to be aimed as a target underneath the V. L. has been reported by Andy (1963). Mundinger and Riechert (1962) aimed the zona incerta by using "Seiten Elektrode".

Narabayashi (1964) reported that V. im. which locates posterior to V. o. p. is in relation to cogwheel phenomenon and its destruction results in selectively diminution of the tremor.

Gillingham (1958) reported a capsulotomy. Smith (1962) has found that in post-mortam examination of a patient in which the fasciculus lenticularis was destructed by stereotaxic operation at the region of posterior limb of the internal capsule and yet he showed complete diminution of the tremor and rigidity without hemiparesis.

Jinnai and Nishimoto (1960), Guiot (1960) mentioned about the thalamocapsulotomy in which the target was the border site of thalamus to the internal capsule.

A substantia nigrolysis (Rand 1959) was also reported as an effective operation.

Recently the destruction of nucleus dentatus has been controversial in the field.

\title{
S-II-2. Past and Present of Method for Stereotactic Lesion
}

\author{
Yuji Mryazaki \\ Department of Neurological Surgery, Sapporo Medical College Hospital
}

It passed 24 years since Spiegel, E. A. and Wycis, H. T. carried out their first case of stereotactic operation for patient of parkinson's disease.

The main research and clinical improvement in stereotactic operation for parkinson's disease in the past 24 years were done to find the target where shows the most effective relief of symptom and for neurophysiological test of expected target in operation theater. On the other hand, as the object of the stereotactic operation is production of localized lesion in the structure of the brain, the final factor of success of this operation is the method of production of artificial lesion in the brain. Many surgeons had paid their effort to get the ideal method.

The author's condition of procedure for production of stereotactic lesion are as follows; 1) Reproducibility, 2) Sharp delimitated lesion, 3) Flexibility, 4) Quick production of lesion, 5) Avascularity, 6) Simplicity, and 7) Reversibility.

The procedure for production of stereotactic lesion done in the past 24 years were divided into 7 groups: 1) Chemical method, 2) Mechanical method, 3) Electrolysis method, 4) Rediofrequency method, 5) Freezing method, 6) Ultrasonic 
method, and 7) Radiation method.

Detailed description on the freezing method was done and the author emphasized that the freezing lesion in the animal brain showed bleeding in and outside of lesion and that several cases expired due to intracerebral bleeding by freezing method in Japan.

As the cooling method has a great advantage as reversible test of target, the permanent lesion should be made by radiofrequency current after reversible lesion in the target by cooling with the same probe.

The method of production of stereotactic lesion used in Japan at present was reported by the data analyzed by the author and the author emphasized that the radiofrequency lesion are used in $80 \%$.

\title{
S-II-3. Operative Indication in the L-DOPA Era
}

\author{
Kenichiro Sugrta \\ Department of Neurosurgery, School of Medicine, Nagoya University
}

Ninety six cases of Parkinsonism have been treated with L-DOPA, in which 46 cases had had no thalamotomy before DOPA treatment. Fourteen cases $(30 \%)$ out of these 46 cases have had thalamotomy because of little effect of DOPA and two other cases were to be operated upon with the same reason. This means the indication of thalamotomy in up to $35 \%$ (16 cases). Since there are cases who have been already selected as the candidate for surgery by neurologist, the actual indication for surgery among the DOPA treated patients goes down to as low as $20 \%$. All these 14 cases had surgery after DOPA treatment for four months. Ten cases had obstinate tremor against DOPA treatment and four showed no effect on DOPA. In twenty four y. o. male DOPA had excellently improved akinesia and rigidity but had to be withdrawn due to hypersexualism. No hypersexualism had been seen after thalamotomy despite resumption of DOPA. 1) The surgical treatment is still necessary. The indication of thalamotomy should be made after at least four months treatment of DOPA. 2) Tremor is most resistant to DOPA therapy. Surgery is indicated most often for these DOPA-resistant tremor. 3) Thalamotomy is now performed mainly to relieve the tremor. Selection of the target point has, therefore, become simpler. However, two of our cases developed hemiparesis on 7 th and 10th postoperative day respectively. Simultaneous treatment of DOPA following surgery might affect higher incidence of such complication, which had been only $2.2 \%$ in the postoperative cases in the pre-DOPA era. Most troublesome side effect of DOPA is the appearance of psychotic symptom. In these cases the same situation might occur even after thalamotomy. 